\title{
High-Throughput Phenotyping of Sorghum Plant Height Using an Unmanned Aerial Vehicle and Its Application to Genomic Prediction Modeling
}

\author{
Kakeru Watanabe1, Wei Guo ${ }^{2}$, Keigo Arai ${ }^{3}$, Hideki Takanashi ${ }^{4}$, Hiromi Kajiya-Kanegae', \\ Masaaki Kobayashi, Kentaro Yano ${ }^{5}$, Tsuyoshi Tokunaga ${ }^{6}$, Toru Fujiwara ${ }^{7}$, \\ Nobuhiro Tsutsumi ${ }^{4}$ and Hiroyoshi Iwata1*
}

'Laboratory of Biometry and Bioinformatics, Department of Agricultural and Environmental Biology, Graduate School of Agricultural and Life Sciences, The University of Tokyo, Tokyo, Japan, ${ }^{2}$ Institute for Sustainable Agro-ecosystem Services, Graduate School of Agricultural and Life Sciences, The University of Tokyo, Tokyo, Japan, ${ }^{3}$ Air4D Co., Ltd., Tokyo, Japan, ${ }^{4}$ Laboratory of Plant Molecular Genetics, Department of Agricultural and Environmental Biology, Graduate School of Agricultural and Life Sciences, The University of Tokyo, Tokyo, Japan, ${ }^{5}$ Bioinformatics Laboratory, Department of Life Sciences, School of Agriculture, Meiji University, Kanagawa, Japan, ${ }^{6}$ Earthnote Co., Ltd., Okinawa, Japan, ${ }^{7}$ Laboratory of Plant Nutrition and Fertilizers, Department of Applied Biological Chemistry, Graduate School of Agricultural and Life Sciences, The University of Tokyo, Tokyo, Japan

\section{OPEN ACCESS}

Edited by: Julie Dickerson, lowa State University, USA

Reviewed by: David Mayerich,

University of Houston, USA Jagadish Rane, Indian Council of Agricultural Research, India

*Correspondence: Hiroyoshi Iwata aiwata@mail.ecc.u-tokyo.ac.jp

Specialty section: This article was submitted to Technical Advances in Plant Science, a section of the journa

Frontiers in Plant Science

Received: 12 January 2017 Accepted: 13 March 2017

Published: 28 March 2017

Citation:

Watanabe K, Guo W, Arai K, Takanashi $H$, Kaijya-Kanegae $H$ Kobayashi M, Yano K, Tokunaga $T$, Fujiwara T, Tsutsumi $N$ and Iwata $H$ (2017) High-Throughput Phenotyping

of Sorghum Plant Height Using an Unmanned Aerial Vehicle and lts Application to Genomic Prediction Modeling. Front. Plant Sci. 8:421. doi: 10.3389/fpls.2017.00421
Genomics-assisted breeding methods have been rapidly developed with novel technologies such as next-generation sequencing, genomic selection and genomewide association study. However, phenotyping is still time consuming and is a serious bottleneck in genomics-assisted breeding. In this study, we established a highthroughput phenotyping system for sorghum plant height and its response to nitrogen availability; this system relies on the use of unmanned aerial vehicle (UAV) remote sensing with either an RGB or near-infrared, green and blue (NIR-GB) camera. We evaluated the potential of remote sensing to provide phenotype training data in a genomic prediction model. UAV remote sensing with the NIR-GB camera and the 50th percentile of digital surface model, which is an indicator of height, performed well. The correlation coefficient between plant height measured by UAV remote sensing ( $\mathrm{PH}_{\cup A V}$ ) and plant height measured with a ruler $\left(\mathrm{PH}_{\mathrm{R}}\right)$ was 0.523. Because PHuAv was overestimated (probably because of the presence of taller plants on adjacent plots), the correlation coefficient between $\mathrm{PH}_{\cup A V}$ and $\mathrm{PH}_{\mathrm{R}}$ was increased to 0.678 by using one of the two replications (that with the lower PHUAV value). Genomic prediction modeling performed well under the low-fertilization condition, probably because PHUAV overestimation was smaller under this condition due to a lower plant height. The predicted values of $\mathrm{PH}_{\cup A V}$ and $\mathrm{PH}_{\mathrm{R}}$ were highly correlated with each other $(r=0.842)$. This result suggests that the genomic prediction models generated with $\mathrm{PH} \cup A v$ were almost identical and that the performance of UAV remote sensing was similar to that of traditional measurements in genomic prediction modeling. UAV remote sensing has a high potential to increase the throughput of phenotyping and decrease its cost. UAV remote sensing will be an important and indispensable tool for high-throughput genomics-assisted plant breeding.

Keywords: genomic prediction, high-throughput phenotyping, near-infrared (NIR), sorghum plant height, UAV remote sensing 


\section{INTRODUCTION}

Improving the throughput of phenotyping in the field is a big challenge in plant genetics, physiology, and breeding. The emergence of the next-generation sequencing technologies enables us to obtain genome-wide DNA polymorphism data for a large number of samples easily and rapidly (Mardis, 2007; Davey et al., 2011). Statistical methods, such as genome-wide association study (GWAS; Brachi et al., 2011; Korte and Farlow, 2013; Huang and Han, 2014) and genomic selection (GS; Meuwissen et al., 2001; Jannink et al., 2010) allow us to associate DNA polymorphism data, which is extremely high-dimensional, to phenotypic variations in agronomic traits. Boosted by these technological developments, the efficiency of plant breeding is expected to improve rapidly (Huang and Han, 2014). However, phenotyping is still time consuming and labor intensive, and may be more costly than genotyping. Thus, phenotyping has become a serious bottleneck in the acceleration of plant breeding (Furbank and Tester, 2011). Field experiments at multiple plant breeding stations over a large geographic area are indispensable to evaluate the adaptability of new candidate genotypes and to examine the pattern of genotype-environment interaction (Chapman et al., 2014). At each breeding station, a large number of genotypes are tested in the field. Most of the measurements conducted in the field are destructive and labor- and time-intensive, and thus cannot be repeated frequently in the course of plant growth. Because phenotypic data is necessary for genomics-assisted breeding, it is the first priority to develop a high-throughput phenotyping method.

Remote sensing using a low-altitude unmanned aerial vehicle (UAV), such as radio-controlled multicopter, can solve the problem described above. Besides low-altitude UAVs, measurements using satellites (Inoue, 1997) and ground-based vehicles (Lee and Searcy, 1999) have been applied for remote sensing of growth conditions of crop plants (Sugiura et al., 2005). However, satellites have low resolution, poor sensitivity under cloudy conditions, and slow data transmission (Sugiura et al., 2005; Zhang and Kovacs, 2012), and ground-based vehicles cannot enter fields with tall crops or muddy soil (Sugiura et al., 2005; Chapman et al., 2014). Low-altitude UAVs have no such disadvantages and can be used without expert skills (Merz and Chapman, 2011). Most low-altitude UAVs have an autopilot function to fly automatically along a route designed by mission planning software (Berni et al., 2009; Chapman et al., 2014; Zarco-Tejada et al., 2014; Díaz-Varela et al., 2015). Another widespread remote sensing technology is light detection and ranging (LiDAR). However, this technology has some shortcomings, e.g., high cost of data acquisition and processing (Díaz-Varela et al., 2015). The emergence of computer-vision technologies, such as the structure-from-motion and multi-viewstereo algorithms, enables reconstruction of accurate 3D-models from a series of images with a considerable overlap between adjacent images. These technologies are attractive alternatives to LiDAR, due to their high performance, flexibility, and relatively low cost (Díaz-Varela et al., 2015). For remote sensing of plants, near-infrared (NIR) cameras have been used in many studies (Lee and Searcy, 1999; Sugiura et al., 2005; Berger et al., 2010;
Cabrera-Bosquet et al., 2011; van Maarschalkerweerd et al., 2013; Colomina and Molina, 2014; Díaz-Varela et al., 2015; TorresSánchez et al., 2015), because plant leaves (or chlorophylls) strongly reflect NIR light (Knipling, 1970; Tucker, 1979; Fahlgren et al., 2015) and some indices based on NIR reflectance rate, such as normalized difference vegetation index (NDVI; Rouse et al., 1974), are useful for identifying plants and assessing their growing conditions via remote sensing. Some studies have indicated that NIR sensors have advantages over standard RGB sensors in plant monitoring (Nijland et al., 2014; Zhang et al., 2016). Nevertheless, NIR cameras are less common, and often more expensive than RGB cameras (or extra cost is needed to modify RGB cameras into NIR cameras). Because remote sensing is a promising tool for phenotyping, we compared the advantages of RGB and NIR cameras in phenotyping and genomic prediction modeling.

Genomic selection is a novel breeding method that allows selection of complex traits with genome-wide markers. Because the selection is performed on the basis of the genetic potential predicted from these markers, GS requires building an accurate prediction model based on a dataset of individuals or lines that have been genotyped and phenotyped (Meuwissen et al., 2001; Jannink et al., 2010). A large dataset is needed to build an accurate prediction model. As mentioned above, however, phenotyping is time consuming and labor intensive, and is a serious bottleneck in building an accurate model. If UAV remote sensing can streamline the collection of phenotypic data, it will greatly enhance the potential of GS.

Using image-processing software for photogrammetry, we can obtain ortho-mosaic and a digital surface model (DSM) from UAV images (Gini et al., 2013). Ortho-mosaic is a distortioncorrected image. DSM provides information on the altitude. In plant science, DSM information has been applied to estimate biomass and plant height of barley (Bendig et al., 2014), and plant height, volume, and canopy size of olive trees (Zarco-Tejada et al., 2014; Díaz-Varela et al., 2015; Torres-Sánchez et al., 2015). Currently, we are using genomics-assisted breeding to develop a sorghum [Sorghum bicolor (L.) Moench] variety that can be used for high bioethanol production for biofuel. Plant height is one of the most important traits affecting bioethanol yield. Because some sorghum accessions may be taller than $4 \mathrm{~m}$, they are usually cut for measurements, which are labor intensive, whereas GS requires phenotypic data and marker genotype data for a large number of accessions.

The objectives of this study are the validation of the usefulness of UAV remote sensing for measurement of sorghum plant height and for genomic prediction modeling. First, we confirmed the accuracy of plant height estimates from UAV images under the conditions of small plot size. Next, we examined the accuracy of genomic prediction of plant height trained by UAV remote sensing data and data manually measured with a ruler. To evaluate the robustness of this method to plant height variation related to environmental differences such as nutrition level, sorghum plants were grown at two levels of nitrogen availability. We also compared the measurement and prediction accuracy of different cameras and different procedures of remote sensing data analysis. 


\section{MATERIALS AND METHODS}

\section{Field Experiment}

In this study, we used 172 accessions from sorghum germplasm collections (Supplementary Table 1). Of these, 78 accessions were from the world core collection of NIAS (National Institute of Agrobiological Science, Ibaraki, Japan; integrated into the National Agriculture and Food Research Organization from April 1, 2016), 91 were from the sorghum mini core collection of ICRISAT (International Crops Research Institute for the Semi-Arid Tropics, Patancheru, India) and 3 were original cultivars developed by EARTHNOTE Co., Ltd. (Okinawa, Japan). Seeds were sown on 200-cell plug trays on May 8, 2014. Seedlings were transplanted to a field of the Institute for Sustainable Agro-ecosystem Services, the University of Tokyo (Tokyo, Japan; $35^{\circ} 44^{\prime} 09.1^{\prime \prime} \mathrm{N}, 139^{\circ} 32^{\prime} 23.7^{\prime \prime} \mathrm{E}, 60 \mathrm{~m}$ above the sea level) on June 9, 2014. An outline of the field design is shown in Figure 1. To investigate the effect of fertilization on plant growth, we used normal (N-P-K: 1.2-1.8-1.6 kg/a) and low (N-P-K: $0.6-1.8-1.6 \mathrm{~kg} / \mathrm{a}$ ) fertilization in two replications per treatment per accession $(172 \times 4=688$ plots in total). Five plants were grown in each plot (inter-plant spacing, $0.3 \mathrm{~m}$; inter-row spacing, $0.72 \mathrm{~m}$ ). On October 2 and 3 , two plants per plot were harvested and their height was measured with a ruler. In total, $688 \times 2=1,376$ plants were measured.

\section{Remote Sensing Experiment}

The radio-controlled quadcopter USM-S1 (Air4D Co., Ltd., Tokyo, Japan; Figure 2A), was used as a UAV for remote sensing. Two digital cameras, Canon PowerShot ELPH 110HS (Canon Inc., Tokyo, Japan), were installed on the UAV (resolution, ca. 16.1 million pixels; sensor size, $6.2 \mathrm{~mm} \times 4.7 \mathrm{~mm}$; focal length, 4.3-21.5 mm). One was a normal RGB camera, and the other one was modified to capture NIR, green and blue (NIR-GB). The NIRGB camera was purchased at MaxMax Inc. (Carlstadt, NJ, USA). The focal length was set at $4.3 \mathrm{~mm}$. The focus was adjusted by the camera auto-focus function. On the ground at each corner of the field, we installed a white acrylic disk (27 $\mathrm{cm}$ in diameter) as a ground control point (GCP) (Figure 2C). The positions of GCPs in the World Geodetic System were measured by using GPS (Geo7X, Trimble Inc., Sunnyvale, CA, USA) and used in image processing.

To compare plant height measured with the UAV ( $\mathrm{PH}_{\mathrm{UAV}}$ ) and plant height measured with rulers $\left(\mathrm{PH}_{\mathrm{R}}\right)$, we performed a remote-sensing experiment on the first day of harvest (October 2). The weather on that day was cloudy. ISO sensitivity, which is an indicator of light sensitivity provided by the International Organization for Standardization (Vernier, Geneva, Switzerland), was set at 320 and shutter speed at $1 / 1,250 \mathrm{~s}$ for the RGB camera; ISO sensitivity was 800 and shutter speed was $1 / 800 \mathrm{~s}$ for the NIR-GB camera. The UAV was controlled by an autopilot system with GPS to fly along a pre-defined course designed by the PC Ground Station software (DJI Co., Ltd., Shenzheng, China). The outline of the flight course is shown in Figure 2B. The course was designed so that the vertical overlap of images was $70 \%$ and horizontal overlap was $30 \%$. Photographs were taken at an altitude of $40 \mathrm{~m}$, total flight time was about $10 \mathrm{~min}$, and 30 photographs were taken. From an altitude of $40 \mathrm{~m}$, the resolution was ca. $13 \mathrm{~mm}$ per pixel. A preliminary remote sensing experiment was performed on July 23 with the RGB camera NEX-7 (Sony Corporation, Tokyo, Japan; resolution, ca. 24 million pixels; sensor size, $23.5 \mathrm{~mm} \times 15.6 \mathrm{~mm}$ ) with a lens of focal length $20 \mathrm{~mm}$. The following parameters were used in the preliminary experiment: ISO sensitivity, 100; shutter speed, 1/800 s; altitude, $50 \mathrm{~m} ; 78$ photographs were taken. From an altitude of $50 \mathrm{~m}$, the resolution was ca. $10 \mathrm{~mm}$ per pixel. Because plants were still small on June 23, we used the data collected on July 23 to obtain DSM data on the ground surface of the field. Although NEX-7 has higher resolution than PowerShot ELPH 110HS, it is heavier and thus we could not mount two types of cameras (RGB and NIR) simultaneously on the UAV. At an altitude of $40 \mathrm{~m}$, the resolution of PowerShot ELPH 110HS was similar to that of

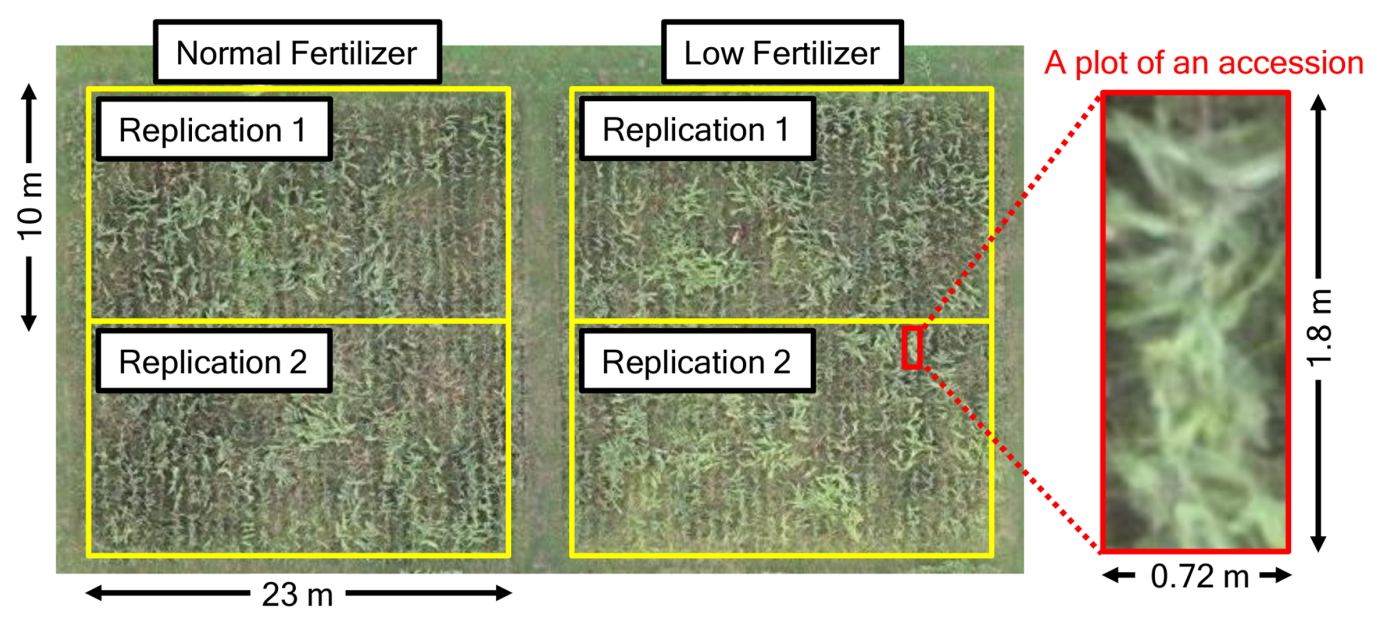

FIGURE 1 | Field design in this study. 


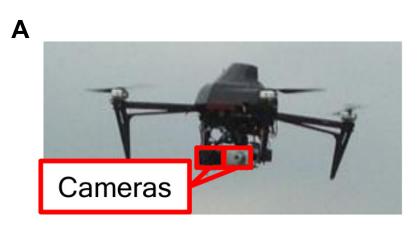

B

C
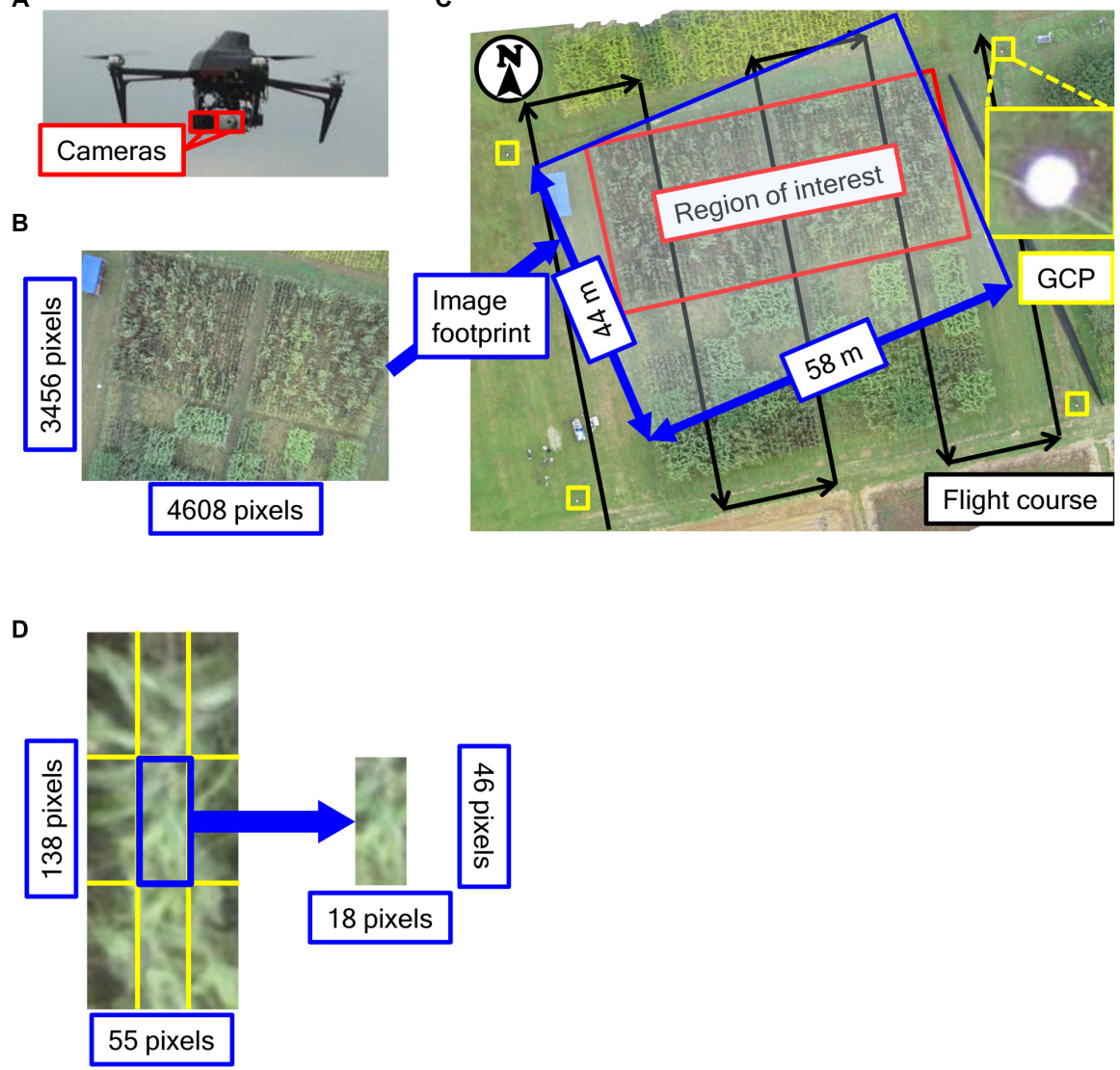

FIGURE 2 | Unmanned aerial vehicle used. (A) Images of UAV used in the study, (B) An image taken in this study, (C) Outline of the flight course and (D) The size of one plot and one divided block. Positions of GCPs in the World Geodetic System were measured by using GPS.

NEX-7. Therefore, we used PowerShot ELPH 110HS for remote sensing.

\section{Image Processing}

Both RGB and NIR-GB images were analyzed in the same way. By using the in-house structure-from-motion software Nadirmetry (Air4D Co., Ltd., Tokyo, Japan), ortho-mosaic images and DSM data were constructed from images taken by the UAV with the geographic coordinates of GCPs. Although the structurefrom-motion algorithm of Nadir-metry is similar to that in other software, it has some advantages in feature point matching and generating point clouds. In the algorithm of Nadir-metry, feature point matching is performed by taking into account the correspondence between overlapping images estimated from their geographic coordinates. As a result, spatial skew hardly occurs. In the generation of point clouds, all pixels were analyzed to detect matching points. This decreased the number of missing matches and prevented point clouds from being sparse. Because DSM values were calculated based on the World Geodetic System 1984 and they did not directly reflect the ground height of objects, we estimated the height of sorghum plants by subtracting the DSM values of the ground surface of the field on July 23 from the DSM values on October 2, as in Bendig et al. (2013). From the location of each plot determined on the ortho-mosaic image, we obtained $\mathrm{PH}_{\mathrm{UAV}}$ for each plot from the DSM data. Because adjacent plots were close to each other, DSMs of plot boundaries were contaminated with data originated from adjacent plots, and might have higher error than those inside a plot. To exclude marginal areas, we divided each plot into 9 blocks $(3 \times 3)$ and analyzed only the central block. That is, the plot size was $0.72 \mathrm{~m} \times 1.8 \mathrm{~m}$ and corresponded to ca. $55 \times 138$ pixels (Figure 2D) at a resolution of $13 \mathrm{~mm}$ per pixel in DSM. The size of the central block was $0.24 \mathrm{~m} \times 0.6 \mathrm{~m}$, corresponding to ca. $18 \times 46$ pixels of DSM (Figure 2D). Each pixel had a DSM value that was construed as the altitude of the location. We calculated the 50th (median), 75th, 90th, and 99th percentiles of DSM values of the central block as the representative values of $\mathrm{PH}_{\mathrm{UAV}}$ for the plot. We evaluated the accuracy of $\mathrm{PH}_{\mathrm{UAV}}$ from its correlation coefficients with $\mathrm{PH}_{\mathrm{R}}$ and also from root mean square difference (RMSD):

$$
\mathrm{RMSD}=\sqrt{\frac{1}{688} \sum_{i=1}^{688}\left(\mathrm{PH}_{\mathrm{UAV}, i}-\mathrm{PH}_{\mathrm{R}, i}\right)^{2}},
$$

where $\mathrm{PH}_{U A V}, i$ and $\mathrm{PH}_{\mathrm{R}}, i$ are the $\mathrm{PH}_{\mathrm{UAV}}$ value and the $\mathrm{PH}_{\mathrm{R}}$ value of the ith plot, respectively. 


\section{Treatment of Unexpected Values}

We assumed that if a plot of a tall accession and that of a small accession were adjacent in the field, the plants of the small accession might be overlapped by those of the tall accession on images, and DSMs of the small accession might be overestimated because they were strongly contaminated by data originated from the tall accession. To confirm this, we compared the correlation between $\mathrm{PH}_{\mathrm{UAV}}$ and $\mathrm{PH}_{\mathrm{R}}$ under three conditions: (1) using data of the replication with higher $\mathrm{PH}_{\mathrm{UAV}}$ value for each accession; (2) using data of the replication with lower $\mathrm{PH}_{\mathrm{UAV}}$ value for each accession; (3) using average $\mathrm{PH}_{\mathrm{UAV}}$ value of two replications for each accession. As described later, the result using the lower $\mathrm{PH}_{\mathrm{UAV}}$ was better than others, and the replication with lower $\mathrm{PH}_{\text {UAV }}$ value for each accession was used in the following analysis.

\section{Genomic Prediction Modeling}

We built genomic prediction models for both $\mathrm{PH}_{\mathrm{UAV}}$ and $\mathrm{PH}_{\mathrm{R}}$, and compared the predicted values. To obtain DNA polymorphism data, we used restriction site-associated DNA sequencing (RAD-Seq; Baird et al., 2008); which is cheaper than whole genome sequencing especially for analysis of DNA polymorphism of large number of accessions. We obtained the data for 66,132 SNPs in 151 accessions (Supplementary Table 1). Genomic best linear unbiased prediction (G-BLUP) using rrBLUP (Endelman, 2011) was used for the modeling. To calculate predicted values for the 151 accessions and compare them for $\mathrm{PH}_{\mathrm{UAV}}$ and $\mathrm{PH}_{\mathrm{R}}$, we performed leave-one-out crossvalidation. In cross-validation, a model built with the $\mathrm{PH}_{\mathrm{UAV}}$ or $\mathrm{PH}_{\mathrm{R}}$ data of 150 of the 151 accessions was used to predict the $\mathrm{PH}_{\mathrm{UAV}}$ or $\mathrm{PH}_{\mathrm{R}}$ values of the remaining accession from its DNA polymorphism data. By comparing the observed and predicted values of both $\mathrm{PH}_{\mathrm{UAV}}$ and $\mathrm{PH}_{\mathrm{R}}$, we evaluated whether manual measurements $\left(\mathrm{PH}_{\mathrm{R}}\right)$ can be replaced with the measurements using UAV remote sensing $\left(\mathrm{PH}_{\mathrm{UAV}}\right)$ in the collection of data for building a model.

\section{RESULTS}

\section{Image Processing and Measurement of Plant Height via UAV Remote Sensing}

For both RGB and NIR-GB cameras, ortho-mosaic images and DSM heat maps of the experimental field before harvest were constructed by using 30 remote-sensing images taken on October 2 (Figure 3).

To assess the accuracy of plant height measurements, we compared the correlation between $\mathrm{PH}_{\mathrm{UAV}}$ and $\mathrm{PH}_{\mathrm{R}}$ obtained with the two cameras at the 50th, 75th, 90th, and 99th percentiles of DSM values (Figure 4). Although there was no significance between two correlation coefficients (RGB vs. NIR-GB) at any combinations, correlation coefficients were higher for the NIRGB than for RGB camera: 0.523 vs. 0.518 (50th percentile), 0.507 vs. 0.504 (75th), and 0.496 vs. 0.491 (90th). However, correlation coefficients were higher for RGB (0.475) than for NIR-GB (0.473) at the 99th percentile. RMSD between $\mathrm{PH}_{U A V}$ and $\mathrm{PH}_{\mathrm{R}}$ was lower for the NIR-GB than RGB camera: (0.649 vs. 0.883 (50th percentile), 0.626 vs. 0.827 (75th), 0.628 vs. 0.792 (90th), and 0.665 vs. 0.759 (99th). $\mathrm{PH}_{\mathrm{UAV}}$ obtained from the RGB camera underestimated $\mathrm{PH}_{\mathrm{R}}$ because the points were distributed below the $y=x$ line in Figure 4. $\mathrm{PH}_{\mathrm{UAV}}$ obtained from the NIR-GB camera estimated $\mathrm{PH}_{\mathrm{R}}$ more accurately, especially at the 90th and 99th percentiles, as evidenced by smaller RMSD in RGB than in NIR-GB.

The relationship between $\mathrm{PH}_{\mathrm{UAV}}$ and $\mathrm{PH}_{\mathrm{R}}$ suggested that $\mathrm{PH}_{\mathrm{UAV}}$ was overestimated at $\mathrm{PH}_{\mathrm{R}}$ values of $<2.0 \mathrm{~m}$ (Figure 4). To assess whether the presence of taller plants on adjacent plots resulted in overestimation, we calculated two types of ratio for each plot as follows:

$$
r_{1}=\frac{\max \left\{\mathrm{PH}_{\mathrm{R}, i, k} \mid k=1,2, \ldots, N\right\}}{\mathrm{PH}_{\mathrm{R}, i}}, r_{2}=\frac{\mathrm{PH}_{\mathrm{UAV}, i}}{\mathrm{PH}_{\mathrm{R}, i}}
$$

where $\mathrm{PH}_{\mathrm{R}, i}$ and $\mathrm{PH}_{\mathrm{UAV}}, i$ are the $\mathrm{PH}_{\mathrm{R}}$ and $\mathrm{PH}_{\mathrm{UAV}}$ values of the ith plot, respectively, and $N=8$ in this case. $\mathrm{PH}_{\mathrm{R}, i, k}$ is the $\mathrm{PH}_{\mathrm{R}}$ value of the $k$ th $(1-8)$ plot adjacent to the $i t h$ plot. The ratio $r_{1}$ represents the degree of height difference among the plants on adjacent plots. The ratio $r_{2}$ represents the degree of overor under- estimation of $\mathrm{PH}_{\mathrm{UAV}}$ against $\mathrm{PH}_{\mathrm{R}}$. A scatter plot of these ratios for one combination (NIR-GB camera and the 50th percentile of DSM values) is shown in Supplementary Figure 1A; the results for other combinations were similar. Theoretically, the $\mathrm{PH}_{\mathrm{UAV}} / \mathrm{PH}_{\mathrm{R}}$ ratio is expected to be approximately constant if UAV measurements are accurate, because both $\mathrm{PH}_{\mathrm{UAV}}$ and $\mathrm{PH}_{\mathrm{R}}$ are expected to be the true values of plant height (with measurement errors). If we regarded $\mathrm{PH}_{\mathrm{R}}$ as the true plant height, the $\mathrm{PH}_{\mathrm{UAV}} / \mathrm{PH}_{\mathrm{R}}$ ratio became large, i.e., the $\mathrm{PH}_{\mathrm{UAV}}$ was overestimated against $\mathrm{PH}_{\mathrm{R}}$ (Supplementary Figure $1 \mathrm{~A}$ ) when $\mathrm{PH}_{\mathrm{R}}$ on adjacent plots was 1.5 times that on the central plot. This result suggests that the presence of taller plants on an adjacent plot prevents accurate construction of the DSM of the target plot because of the overlapping effect.

We analyzed the relationships between $\mathrm{PH}_{U A V}$ and $\mathrm{PH}_{\mathrm{R}}$ for all the combinations of the two cameras and four percentile values separately for each of two replications (one with the lower $\mathrm{PH}_{\mathrm{UAV}}$ value, the other one with the higher $\mathrm{PH}_{\mathrm{UAV}}$ value) and the average (Figure 5). For all combinations, the correlation coefficients were highest (around 0.65) for the replications with lower $\mathrm{PH}_{\mathrm{UAV}}$ values and lowest (around 0.40) for the replications with higher $\mathrm{PH}_{\mathrm{UAV}}$ values. Correlation coefficients with lower $\mathrm{PH}_{\mathrm{UAV}}$ were significantly higher than those with mean $\mathrm{PH}_{\mathrm{UAV}}$ at significant level of $10 \%$ at all combinations. This result suggested that the lower $\mathrm{PH}_{\mathrm{UAV}}$ values were more reliable.

\section{Genomic Prediction Modeling}

To evaluate the accuracy of genomic prediction for $\mathrm{PH}_{\mathrm{UAV}}$ and $\mathrm{PH}_{\mathrm{R}}$ and the agreement between their predicted values, we obtained these values via leave-one-out cross- validation for all sorghum accessions cultivated under normal and low fertilization conditions. The relationships between the observed and predicted $\mathrm{PH}_{\mathrm{UAV}}$ and $\mathrm{PH}_{\mathrm{R}}$ values are shown in Figure 6. If the combination of two cameras and four representative values is different, the combination 

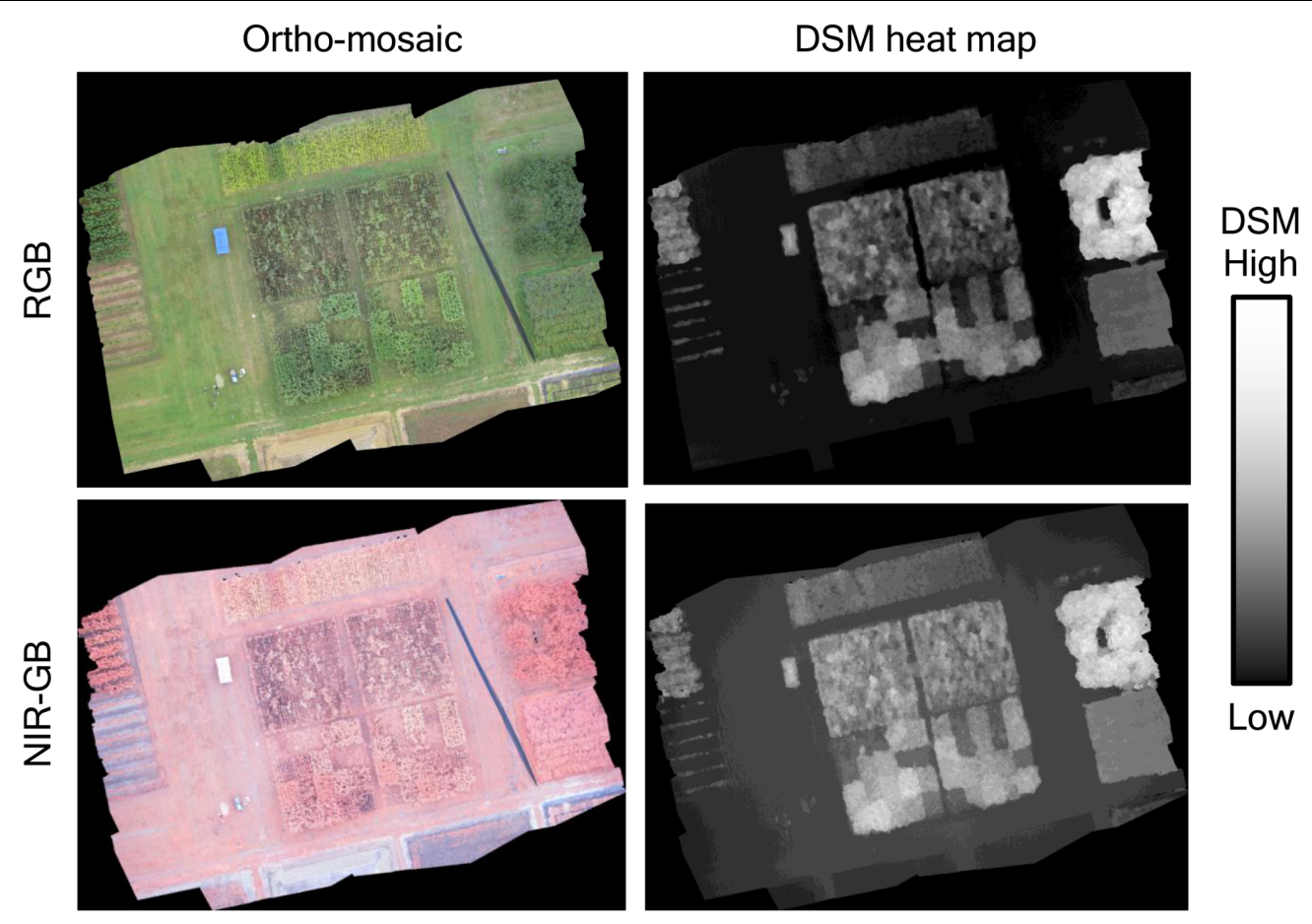

FIGURE 3 | Ortho-mosaic and DSM heat map of October 2. Upper images were originated from the standard RGB cameras and lower images were originated from the NIR-GB (near-infrared, green, blue) camera. DSM provides information of the altitude. The resolution was ca. 13 mm per pixel. The sizes of both the ortho-mosaic and DSM were 10,701 × 8,061 pixels in RGB and 10,749 × 8,041 in NIR-GB; the ortho-mosaic and DSM were built from 30 images $(4,608 \times 3,456$ pixels each).

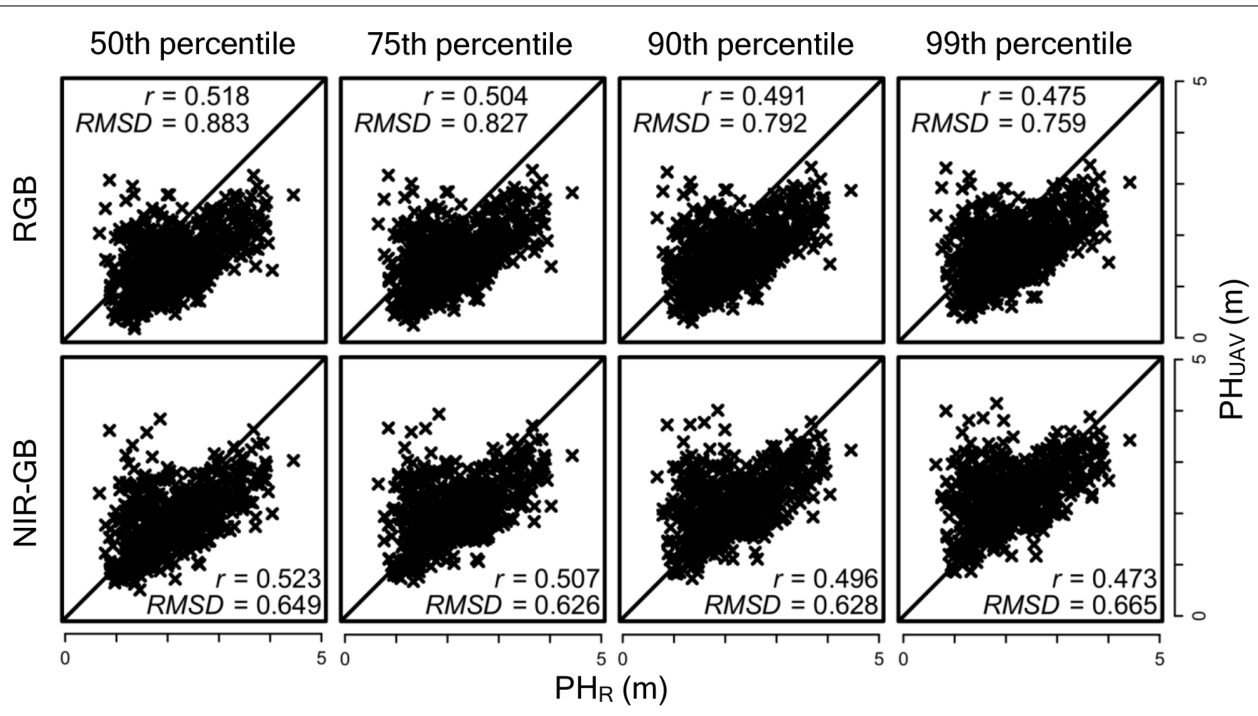

FIGURE 4 | Accuracy under different cameras and different percentiles of DSM. The diagonal line indicates $y=x$; $r$ correlation coefficient; RMSD, root mean

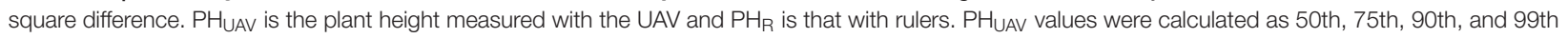
percentile of DSM values in each plot.

of selected replications for each fertilization condition is also different. For example, we consider two combinations: (i) $\left(\mathrm{PH}_{\mathrm{UAV}}\right.$ of replication 1) $<\left(\mathrm{PH}_{\mathrm{UAV}}\right.$ of replication 2$)$ for accession $\mathrm{A}$ and $\left(\mathrm{PH}_{\mathrm{UAV}}\right.$ of replication 1$)<\left(\mathrm{PH}_{\mathrm{UAV}}\right.$ of replication 2) for accession $B$ in a combination of two cameras and four percentiles of DSM values (combination 1), and (ii) $\left(\mathrm{PH}_{\mathrm{UAV}}\right.$ of replication 1$)<\left(\mathrm{PH}_{\mathrm{UAV}}\right.$ of replication 2$)$ for accession $\mathrm{A}$ and $\left(\mathrm{PH}_{\mathrm{UAV}}\right.$ of replication 1) $>\left(\mathrm{PH}_{\mathrm{UAV}}\right.$ 

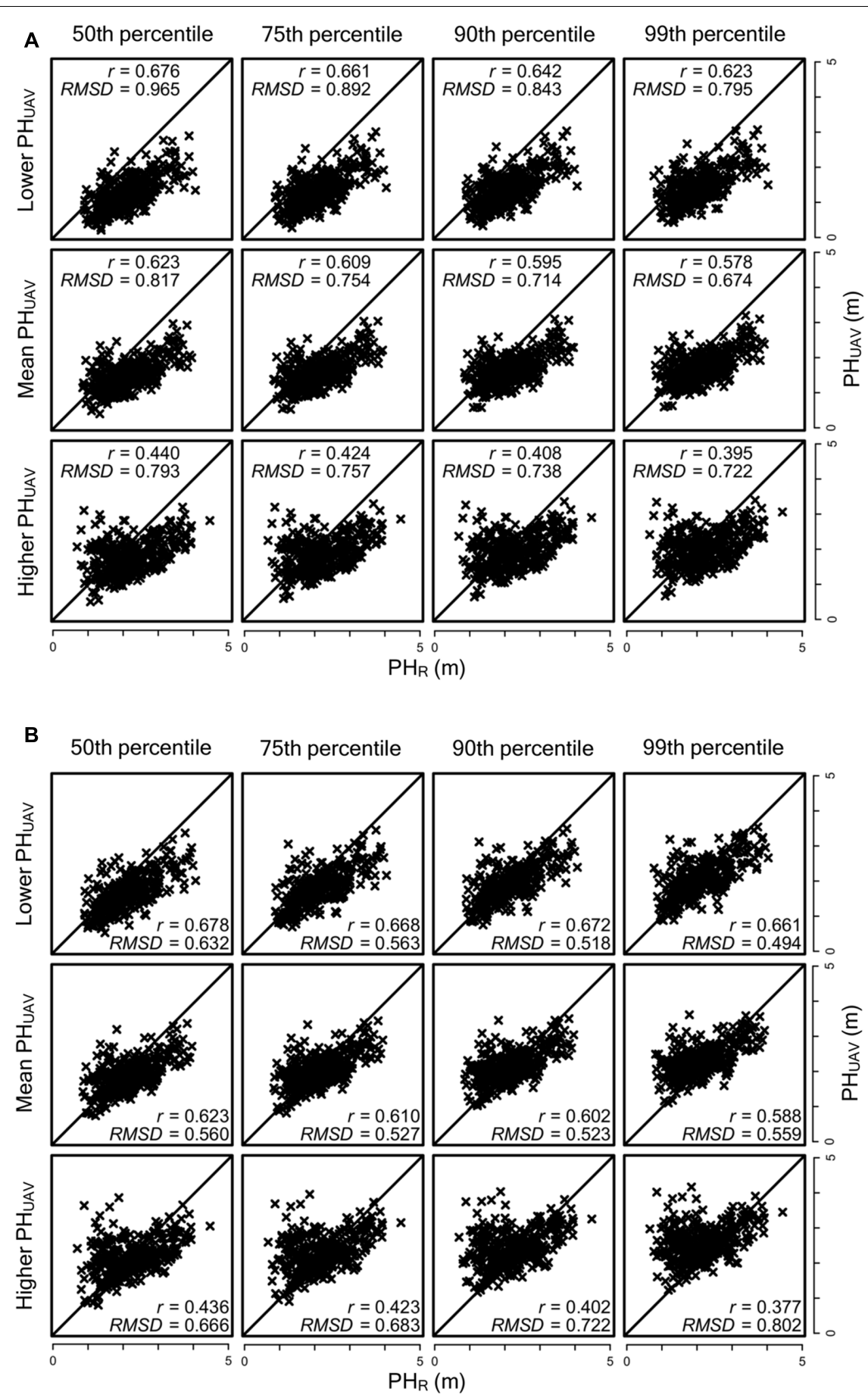

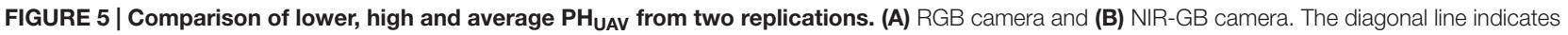
$y=x ; r$, correlation coefficient; RMSD, root mean square difference.

of replication 2) for accession $B$ in another combination (combination 2). Then, for accession A, replication 1 is selected as the plot with the lower $\mathrm{PH}_{\mathrm{UAV}}$ value in both combinations, whereas for accession B replication 1 is selected in combination 1 and replication 2 is selected in combination 2 as the plot with lower $\mathrm{PH}_{U A V}$ value. The same combinations of replications were also used for $\mathrm{PH}_{\mathrm{R}}$. However, the combinations of replications were almost the same between different combinations of two cameras and four percentiles. Because of this, the results of $\mathrm{PH}_{\mathrm{R}}$ prediction with different cameras and different percentiles were similar to each other. The combinations with the highest and the lowest correlation coefficients are shown in Figure 6 


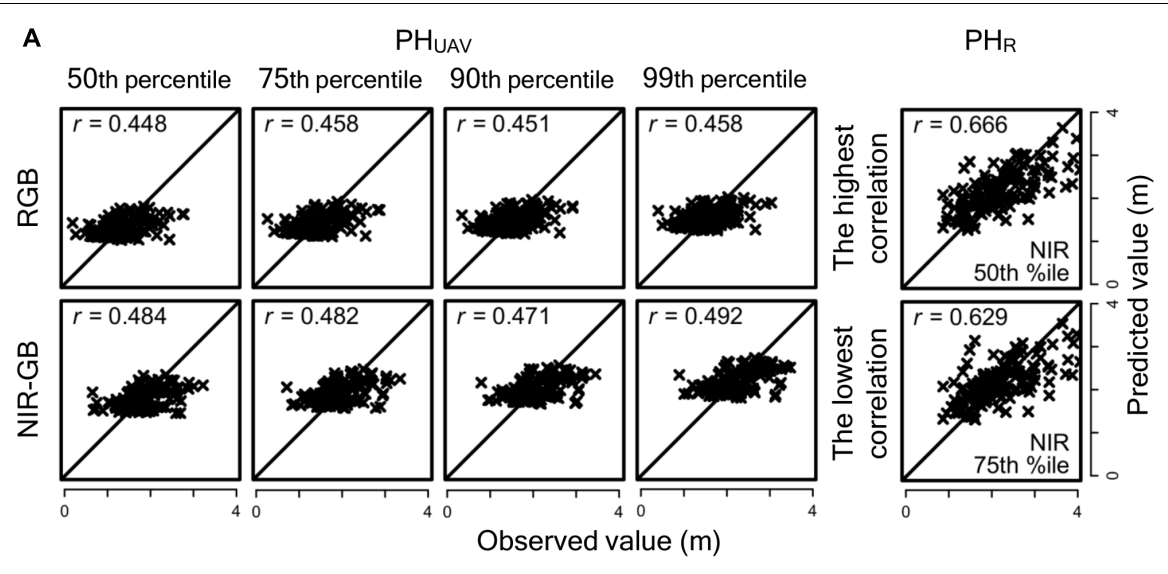

B

PHUAV
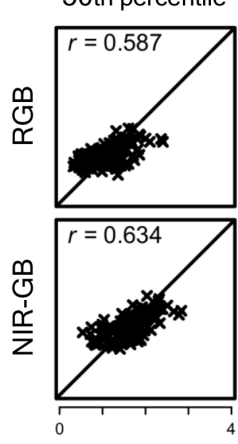

75th percentile
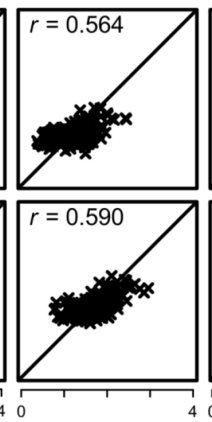
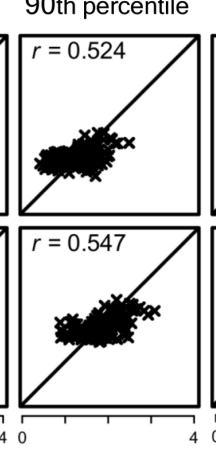

Observed value $(\mathrm{m})$
$\mathrm{PH}_{\mathrm{R}}$

99th percentile
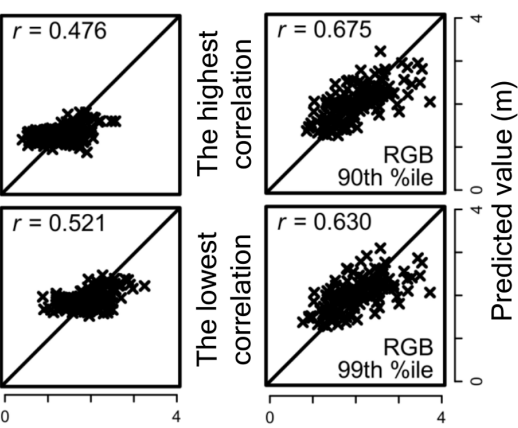

FIGURE 6 | Relationships between observed and predicted $\mathrm{PH}_{\mathrm{UAV}}$ and $\mathrm{PH}_{\mathbf{R}}$ values in genomic prediction under normal and low fertilization conditions. (A) Normal fertilization and (B) Low fertilization. $r$, correlation coefficient. For $\mathrm{PH}_{\mathrm{R}}$, only the combinations the highest and lowest correlation coefficients are shown.

for $\mathrm{PH}_{\mathrm{R}}$ prediction. Under normal fertilization, correlation coefficients between observed and predicted $\mathrm{PH}_{\mathrm{UAV}}$ were less than 0.5 (range, 0.448-0.492) for all combinations of the type of cameras and the percentiles of DSM, whereas those for $\mathrm{PH}_{\mathrm{R}}$ ranged from 0.629 to 0.675 . Correlation coefficients for $\mathrm{PH}_{\mathrm{UAV}}$ were higher under low fertilization than under normal fertilization in all combinations. Although almost all combinations had lower correlation coefficients for PHUA than for $\mathrm{PH}_{\mathrm{R}}$, the correlation coefficient for $\mathrm{PH}_{\mathrm{UAV}}$ in the combination of NIR-GB and 50th percentile was as high as that for $\mathrm{PH}_{\mathrm{R}}$ (Figure 6). Predicted $\mathrm{PH}_{\mathrm{UAV}}$ and predicted $\mathrm{PH}_{\mathrm{R}}$ highly correlated with each other in all combination, (correlation coefficients $\geq 0.66$ under low fertilization and $\geq 0.78$ under normal fertilization except for one combination; Figure 7).

\section{DISCUSSION}

We introduced UAV remote sensing for high-throughput measurement of sorghum plant height, and applied it to genomic prediction modeling. The results of this study suggest the potential of UAV remote sensing for the highthroughput phenotyping of plant height in sorghum. Some sorghum genotypes are too tall to measure the height of the plants without harvesting them. Our approach would not only decrease labor cost but would also allow observation of plant growth over time. Traditionally, all accessions are measured only once, although their growth stages at the measurement time may differ. Our remote sensing approach would allow comparison of accessions at the same growth stage.

Using UAV remote sensing, we could not measure sorghum plant height as accurately as that of barley (which is smaller than sorghum) measured by Bendig et al. (2014). One reason may be that tall sorghum plants overlap (Supplementary Figure 1). Another reason may be low plant density: 3.9 plants $/ \mathrm{m}^{2}$ vs. 300 plants $/ \mathrm{m}^{2}$ in Bendig et al. (2014); we can see the sparseness of plants in a plot in Figure 1. If plant density is high enough to form a continuous canopy, most of the matched points are captured from the canopy and DSM reflects plant height precisely. However, because of the sparseness, matched points included not only the top of canopy but also the ground or lower parts of plants. This could cause errors on 


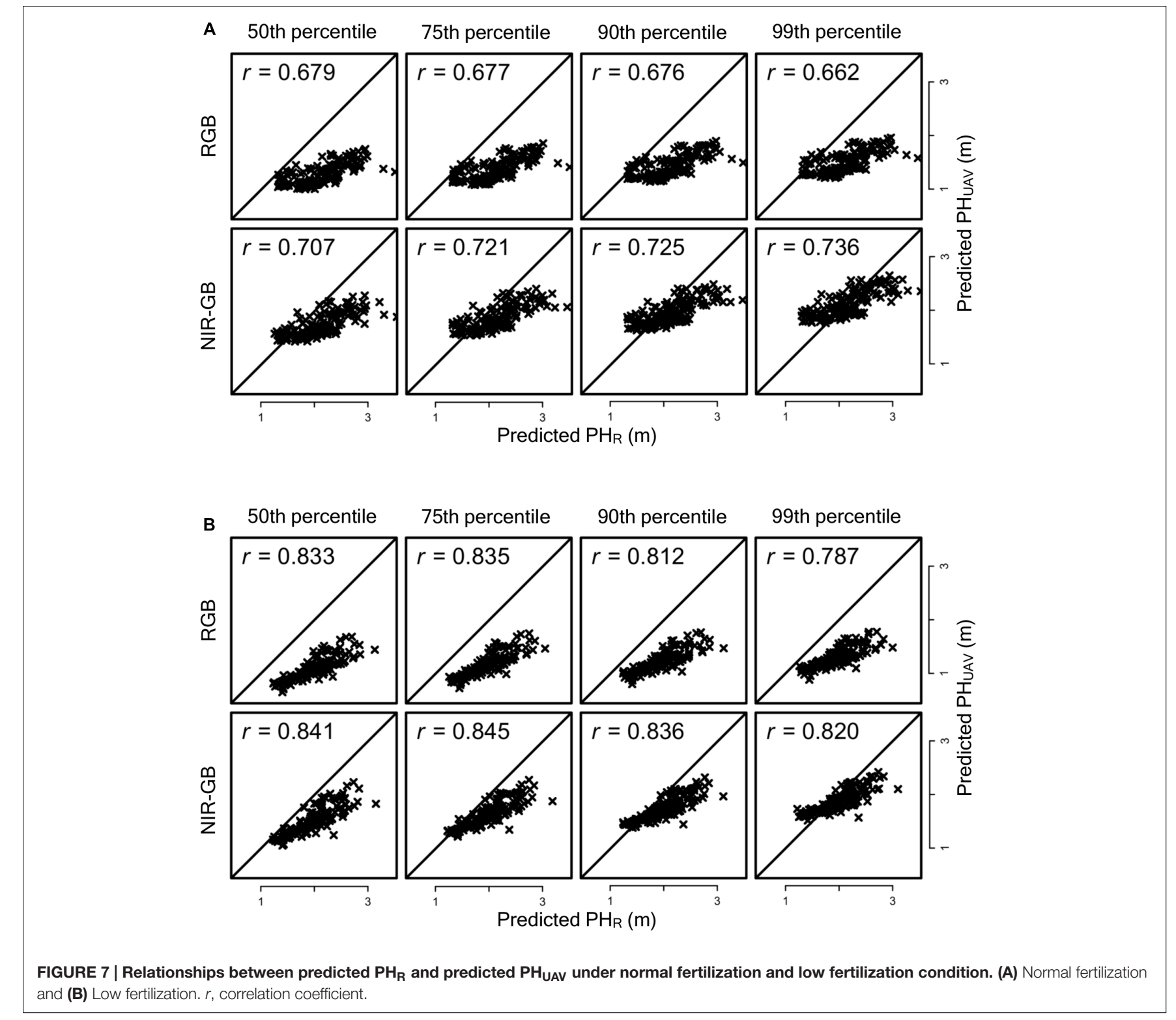

DSM in plant height measurement. Increasing plot size and plant density may improve the measurement accuracy. We can easily measure plant height multiple times with UAV remote sensing, which would probably also reduce measurement error. An important point is that the predicted $\mathrm{PH}_{\mathrm{UAV}}$ values were highly correlated with the predicted values of $\mathrm{PH}_{\mathrm{R}}$, even though the correlation between the observed values of $\mathrm{PH}_{\mathrm{UAV}}$ and $\mathrm{PH}_{\mathrm{R}}$ was not high. This is because the observed values of $\mathrm{PH}_{\mathrm{UAV}}$ and $\mathrm{PH}_{\mathrm{R}}$ had different types of errors (e.g., errors from manual measurements and UAV measurements). Higher correlation between the predicted values suggests that they were less affected by errors than observed values. If we can reduce the measurement errors of UAV remote sensing by improving technologies or by repeating measurements, UAV remote sensing will perform better than manual measurements for genomicsassisted breeding.
We also found that using the lower value of $\mathrm{PH}_{\mathrm{UAV}}$ of the two replications for each association seemed to reduce the overlapping effect (Figure 5). For the replications with the lower value $\mathrm{PH}_{\mathrm{UAV}}$ values, the correlation coefficients of $\mathrm{PH}_{\mathrm{UAV}}$ and $\mathrm{PH}_{\mathrm{R}}$ were not largely different for the RGB and NIR-GB cameras. The RGB camera underestimated $\mathrm{PH}_{\mathrm{UAV}}$ and the RMSD value of this camera was higher than that of the NIR-GB camera. For genomic prediction modeling, the correlation coefficients between observed and predicted values were higher for NIR-GB than RGB (Figures 6, 7), indicating that NIR-GB was slightly superior for this purpose. Only the combination of NIR-GB and 50th percentile under low fertilization resulted in a correlation coefficient between observed and predicted values for $\mathrm{PH}_{\mathrm{UAV}}$ close to that for $\mathrm{PH}_{\mathrm{R}}$ (Figure 6). Prediction of plant height was less accurate under normal than under low fertilization. This may suggest that the overlapping effect was stronger when plants 
were larger. NIR-GB was better than RGB under both fertilization conditions. Like in crop identification (Zhang et al., 2016) and in monitoring of plant condition (Nijland et al., 2014), a NIR sensor may perform better than a standard RGB sensor for remote sensing in plant breeding.

In the case of the NIR-GB camera, the correlation coefficient was the highest at the 50th percentile of DSM values, whereas the RMSD value was the lowest at the 99th percentile. At the 99th percentile, $\mathrm{PH}_{\mathrm{UAV}}$ was almost the same as $\mathrm{PH}_{\mathrm{R}}$. RMSD became higher, but the number of outliers also increased, which reduced the correlation coefficient. At the 50th percentile, the number of outliers was low in $\mathrm{PH}_{\mathrm{UAV}}$ measurements and the correlation coefficient became higher, but the difference between the $\mathrm{PH}_{\mathrm{UAV}}$ and $\mathrm{PH}_{\mathrm{R}}$ values increased and RMSD became lower. There is probably a trade-off between the correlation coefficient and RMSD. If accurate plant height measurements are required, $\mathrm{PH}_{\mathrm{UAV}}$ at the 99th percentile of DSM will perform well. In this study, $\mathrm{PH}_{\mathrm{UAV}}$ at the 50th percentile of DSM was better than at other percentile values regarding the accuracy of both plant height measurements and genomic prediction modelings.

The collection of $\mathrm{PH}_{\mathrm{UAV}}$ data required 3 people $\times 10 \mathrm{~min}$, while collection of $\mathrm{PH}_{\mathrm{R}}$ data required over 10 people $\times 2$ days. Because GS and GWAS require phenotyping of a large number of accessions or plants, UAV remote sensing will be an important and indispensable tool for high-throughput genomic-assisted plant breeding.

Using digital cameras, we can measure canopy cover from images taken right above the plants (Purcell, 2000) and relate it to plant density, early vigor, leaf size, and radiation interception (Liebisch et al., 2015). Using NIR sensors, we can measure NDVI of the canopy and relate it to canopy biomass and nitrogen status (Hansen and Schjoerring, 2003). Using thermal sensors, we can measure canopy temperature (Berni et al., 2009) and relate it to water stress (Jackson et al., 1981).

Not all of the studies used UAV remote sensing. However, by attaching appropriate sensors to an UAV, we can obtain various types of information from plants grown in the field and measure important target trait-related characteristics. Combination of

\section{REFERENCES}

Baird, N. A., Etter, P. D., Atwood, T. S., Currey, M. C., Shiver, A. L., Lewis, A. L., et al. (2008). Rapid SNP discovery and genetic mapping using sequenced RAD markers. PLoS ONE 3:e3376. doi: 10.1371/journal.pone. 0003376

Bendig, J., Bolten, A., Bennertz, S., Broscheit, J., Eichfus, S., and Bareth, G. (2014). Estimation biomass of barley using crop surface models (CMSs) derived from UAV-based RGB imaging. Remote Sens. 6, 10395-10412. doi: 10.3390/ rs61110395

Bendig, J., Bolton, A., and Bareth, G. (2013). UAV-based imaging for multitemporal, very high resolution crop surface models to monitor crop growth variability. PFG. 6, 551-562.

Berger, B., Parent, B., and Tester, M. (2010). High-throughput shoot imaging to study drought response. J. Exp. Bot. 261, 3519-3528. doi: 10.1093/jxb/ erq201

Berni, J. A. J., Zarco-Tejada, P. J., Suárez, L., and Fereres, E. (2009). Thermal and narrowband multispectral remote sensing for vegetation monitoring from machine learning and image analysis enables the evaluation of complex traits, such as flowering date (Guo et al., 2015). In the future, various kinds of plant phenotyping data will be measured in parallel and in a high-throughput manner by UAV remote sensing.

\section{AUTHOR CONTRIBUTIONS}

KW, TF, NT, and HI planned and designed the experiments. TT and NT contributed to the preparation of materials. KW, WG, and KA performed remote sensing experiments. HT performed the RAD-Seq experiment, and MK, HK-K, and KY analyzed the RAD-Seq data. KW performed other analyses, interpreted results, and drafted the manuscript with the contributions from WG, KA, and HI. NT obtained funding for the study. HI supervised the study. All authors read and approved the final manuscript.

\section{FUNDING}

The research was funded by CREST, Japan Science and Technology Agency, Japan (research subject: "Novel techniques of tailor-made breeding for energy crop improvement using high-throughput genotyping").

\section{ACKNOWLEDGMENTS}

The authors thank the technical support staff at the Institute of Sustainable Agro-ecosystem Services, The University of Tokyo for their help in field experiments.

\section{SUPPLEMENTARY MATERIAL}

The Supplementary Material for this article can be found online at: http://journal.frontiersin.org/article/10.3389/fpls.2017.00421/ full\#supplementary-material

an unmanned aerial vehicle. IEEE Trans. Geosci. Remote Sens. 47, 722-738. doi: 10.1109/TGRS.2008.2010457

Brachi, B., Morris, G. P., and Borevitz, J. O. (2011). Genome-wide association studies in plants: the missing heritability is in the field. Genome Biol. 12:232. doi: 10.1186/gb-2011-12-10-232

Cabrera-Bosquet, L., Moloero, G., Stellacci, A. M., Bort, J., Nogués, S., and Araus, J. L. (2011). NDVI as a potential tool for predicting biomass, plant nitrogen content and growth in wheat genotypes subjected to different water and nitrogen conditions. Cereal Res. Commun. 39, 147-159. doi: 10.1556/CRC.39. 2011.1.15

Chapman, S., Merz, T., Chan, A., Jackway, P., Hrabar, S., Dreccer, M. F., et al. (2014). Pheno-copter: a low-altitude, autonomous remote-sensing robotic helicopter for high-throughput field-based phenotyping. Agronomy 4, 279-301. doi: 10.3390/agronomy4020279

Colomina, I., and Molina, P. (2014). Unmanned aerial systems for photogrammetry and remote sensing: a review. ISPRS J. Photogramm. Remote Sens. 92, 79-97. doi: 10.1016/j.isprsjprs.2014. 02.013 
Davey, J. W., Hohenlohe, P. A., Etter, P. D., Boone, J. Q., Catchen, J. M., and Blaxter, M. L. (2011). Genome-wide genetic marker discovery and genotyping using next-generation sequencing. Nat. Rev. Genet. 12, 499-510. doi: 10.1038/nrg3012

Díaz-Varela, R. A., de la Rosa, R., León, L., and Zarco-Tejata, P. J. (2015). High-resolution airborne UAV imagery to assess olive tree crown parameters using 3D photo reconstruction: application in breeding trials. Remote Sens. 7 , 4213-4232. doi: 10.3390/rs70404213

Endelman, J. B. (2011). Ridge regression and other kernels for genomic selection with R package rrBLUP. Plant Genome 4, 250-255. doi: 10.3835/ plantgenome2011.08.0024

Fahlgren, N., Gehan, M. A., and Baxter, I. (2015). Lights, camera, action: highthroughput plant phenotyping is ready for a close-up. Curr. Opin. Plant Biol. 24, 93-99. doi: 10.1016/j.pbi.2015.02.006

Furbank, R. T., and Tester, M. (2011). Phenomics-technologies to relieve the phenotyping bottleneck. Trends Plant Sci. 16, 635-644. doi: 10.1016/j.tplants. 2011.09.005

Gini, R., Pagliari, D., Passoni, D., Pinto, L., Sona, G., and Dosso, P. (2013). UAV photogrammetry: block triangulation comparisons. Int. Arch. Photogram. Remote Sens. Spat. Inf. Sci. Volume XL-1/W2, 157-162. doi: 10.5194/ isprsarchives-XL-1-W2-157-2013

Guo, W., Fukatsu, T., and Ninomiya, S. (2015). Automated characterization of flowering dynamics in rice using field-acquired time-series RGB images. Plant Methods 11:7. doi: 10.1186/s13007-015-0047-9

Hansen, P. M., and Schjoerring, J. K. (2003). Reflectance measurement of canopy biomass and nitrogen status in wheat crops using normalized difference vegetation indices and partial least square regression. Remote Sens. Environ. 86, 542-553. doi: 10.1016/S0034-4257(03)00131-7

Huang, X., and Han, B. (2014). Natural variation and genome-wide association studies in crop plants. Ann. Rev. Plant Biol. 65, 531-551. doi: 10.1146/annurevarplant-050213-035715

Inoue, Y. (1997). Remote sensing of crop and vegetative environment (Part 2). J. Japan Remote Sens. Soc. 17, 57-67.

Jackson, R. D., Idso, S. B., Reginato, R. J., and Pinter, P. J. Jr. (1981). Canopy temperature as a crop water stress indicator. Water Resour. Res. 17, 1133-1138. doi: 10.1029/WR017i004p01133

Jannink, J. L., Lorenz, A. J., and Iwata, H. (2010). Genomic selection in plant breeding: from theory to practice. Brief. Funct. Genomics 9, 166-177. doi: 10. 1093/bfgp/elq001

Knipling, E. B. (1970). Physical and physiological basis for the reflectance of visible and near-infrared radiation from vegetation. Remote Sens. Environ. 1, 155-159. doi: 10.1016/S0034-4257(70)80021-9

Korte, A., and Farlow, A. (2013). The advantage and limitations of trait analysis with GWAS: a review. Plant Methods 9:29. doi: 10.1186/1746-4811-9-29

Lee, W., and Searcy, S. (1999). "Multispectral sensor for detecting nitrogen in corn plants," in Proceedings of the 2000 ASAE Annual International Meeting. Paper No. 001010, Milwaukee, WI.

Liebisch, F., Kirchgessner, N., Schneider, D., Walter, A., and Hund, A. (2015). Remote, aerial phenotyping of maize traits with a mobile multi-sensor approach. Plant Methods 11:9. doi: 10.1186/s13007-015-0048-8

Mardis, E. R. (2007). The impact of next-generation sequencing technology on genetics. Trends Genet. 24, 133-141. doi: 10.1016/j.tig.2007.12.007

Merz, T., and Chapman, S. (2011). “Autonomous unmanned helicopter system for remote sensing missions in 655 unknown environments," in Proceedings of the International Conference on Unmanned Aerial Vehicle 656 in Geomatics, (Zurich: ISPRS).

Meuwissen, T. H., Hayes, B. J., and Goddard, M. E. (2001). Prediction of total genetic value using genome-wide dense marker maps. Genetics 157, 1819-1829.

Nijland, W., de Jong, R., de Jong, S. M., Wulder, M. A., Bater, C. W., and Coops, N. C. (2014). Monitoring plant condition and phenology using infrared sensitive consumer grade digital cameras. Agric. For. Meteorol. 184, 98-106. doi: 10.1016/j.agrformet.2013.09.007

Purcell, L. C. (2000). Soybean canopy coverage and light interception measurements using digital imagery. Crop Sci. 40, 834-837. doi: 10.2135/ cropsci2000.403834x

Rouse, J. W., Haas, R. H., Schell, J. A., and Deering, D. W. (1974). "Monitoring vegetation systems in the Great Plains with ERTS," in Proceedings of the Third ERTS-1 Symposium, NASA SP-351, (Washington, DC: NASA), 309-317.

Sugiura, R., Noguchi, N., and Ishii, K. (2005). Remote-sensing technology for vegetation monitoring using an unmanned helicopter. Biosyst. Eng. 90, 369-379. doi: 10.1016/j.biosystemseng.2004.12.011

Torres-Sánchez, J., López-Granados, F., Serrano, N., Arquero, O., and Peña, J. M. (2015). High-throughput 3-D monitoring of agricultural-tree plantations with unmanned aerial vehicle (UAV) technology. PLOS ONE 10:e0130479. doi: 10. 1371/journal.pone.0130479

Tucker, C. J. (1979). Red and photographic infrared linear combinations for monitoring vegetation. Remote Sens. Environ. 8, 127-150. doi: 10.1016/00344257(79)90013-0

van Maarschalkerweerd, M., Bro, R., Egebo, M., and Husted, S. (2013). Diagnosing latent copper deficiency in intact barley leaves (Hordeum vulgare, L.) using near infrared spectroscopy. J. Agric. Food Chem. 61, 10901-10910. doi: 10.1021/ jf402166g

Zarco-Tejada, P. J., Diaz-Varela, R., Angileri, V., and Loudjani, P. (2014). Tree height quantification using very high resolution and imagery acquired from an unmanned aerial vehicle (UAV) and 3D photo-reconstruction methods. Eur. J. Agronomy 55, 89-99. doi: 10.1016/j.eja.2014.01.004

Zhang, C., and Kovacs, J. M. (2012). The application of small unmanned aerial system for precision agriculture: a review. Precis. Agric. 13, 693-712. doi: 10. 1007/s11119-012-9274-5

Zhang, J., Yang, C., Song, H., Hoffmann, W. C., Zhang, D., and Zhang, G. (2016). Evaluation of an airborne remote sensing platform consisting of two consumer-grade cameras for crop identification. Remote Sens. 8:257. doi: 10. $3390 /$ rs8030257

Conflict of Interest Statement: The authors declare that the research was conducted in the absence of any commercial or financial relationships that could be construed as a potential conflict of interest.

Copyright (c) 2017 Watanabe, Guo, Arai, Takanashi, Kajiya-Kanegae, Kobayashi, Yano, Tokunaga, Fujiwara, Tsutsumi and Iwata. This is an open-access article distributed under the terms of the Creative Commons Attribution License (CC BY). The use, distribution or reproduction in other forums is permitted, provided the original author(s) or licensor are credited and that the original publication in this journal is cited, in accordance with accepted academic practice. No use, distribution or reproduction is permitted which does not comply with these terms. 